\title{
Urgencias hospitalarias y extrahospitalarias en Navarra. Razones que las motivan
}

\section{Reasons for hospital and ambulatory emergencies in Navarre}

\author{
I. Pérez-Ciordia ${ }^{1}$, F. Guillén Grima ${ }^{2,3}$
}

\section{RESUMEN}

Fundamento. La demanda de asistencia sanitaria urgente está alcanzando cifras cercanas a la saturación del sistema. El objetivo del trabajo es describir el perfil del demandante de urgencias hospitalarias y extrahospitalarias en Navarra y conocer los factores que motivan la utilización de los servicios de urgencias.

Metodología. Estudio multicéntrico transversal. Se utilizó un cuestionario autoadministrado de respuestas múltiples. Se entrevistaron a 2.364 pacientes que acudieron a un servicio de urgencias hospitalario o extrahospitalario (excluidos las visitas domiciliarias) en Navarra, entre el 15 y el 21 de noviembre de 2007.

Se recogen datos descriptivos del paciente, razones que le motivan a solicitar asistencia urgente agrupadas en 3 bloques (18 ítems) y sensación de gravedad (leve, moderada, grave) que otorga a su sintomatología.

La asociación entre autopercepción de gravedad y acudir a hospital y las otras variables de estudio se analizó mediante regresión logística no condicional.

Resultados. La atención extrahospitalaria es la más demandada (62,7\%). Las razones principales para usar los servicios son necesidad (66,3\%) y comodidad (40,7\%). Existen diferencias estadísticamente significativas entre las demandas hospitalaria y extrahospitalaria. Quienes valoran su estado como leve $(24,1 \%)$, acuden más a su servicio extrahospitalaria (OR:1,4; IC95\%:1,1-1,9), su estado de salud habitual es bueno (OR:2,1; IC95\%:1,3-3,2) y señalan razones de comodidad (OR:1,6; IC95\%:1,3-2) como razones de demanda.

Conclusiones. Los usuarios y los médicos deben participar en el debate para intentar incidir en el mal uso de los servicios sanitarios, tanto por exceso como por defecto.

Palabras clave. Utilización servicios de urgencias. Necesidades. Motivos de utilización.

\begin{abstract}
Background. The demand for emergency health care is reaching figures that are close to saturating the system. The aim of the paper is to describe the profile of the user of hospital and ambulatory emergency care in Navarre and to determine the factors that motivate use of the emergency services.
\end{abstract}

Methodology. Multicentric transversal study. A self-administered questionnaire with multiple answers was employed. Two thousand three hundred and sixty-four patients who attended a hospital or ambulatory emergency service (excluding home visits) in Navarre between November $15^{\text {th }}$ and $21^{\text {st }} 2007$.

We collected descriptive data on the patient, reasons for requesting emergency care grouped into three blocks (18 items) and sensation of seriousness (light, moderate, serious) that he/she attributes to his/her symptomology.

The association between self-perception of seriousness and going to hospital and the other variables studied was analysed through non-conditional logistic regression.

Results. The highest demand was for ambulatory care $(62.7 \%)$. The principal reasons for using the services are need $(66.3 \%)$ and convenience $(40.7 \%)$. There are statistically significant differences between hospital and outpatient demands. Those who evaluate their state as light (24.1\%) attend ambulatory accidents and emergencies more (OR:1.4; CI95\%:1.1-1.9), have a state of health that is normally good (OR:2.1; CI95\%:1.3-3.2), and indicate reasons of convenience (OR:1.6; CI95\%:1.3-2) as reasons for the demand.

Conclusions. Both users and doctors should participate in the debate to try and influence the inappropriate use of the health services, both through excess and by default.

Key words. Use of accident and ambulatory services. Needs. Reasons for use.
1. Servicio de Urgencias Extrahospitalarias. Centro de Salud de Tafalla. Navarra.

2. Unidad de Medicina Preventiva. Clínica Universidad de Navarra.

3. Departamento de Ciencias de la Salud. Universidad Pública de Navarra.

\section{Correspondencia}

Ignacio Pérez-Ciordia

Servicio de Urgencias Extrahospitalarias

Centro de Salud de Tafalla. Navarra

Tfno. 948704034

E-mail: ignacio_perez_ciordia@hotmail.com 


\section{INTRODUCCIÓN}

La demanda de asistencia sanitaria urgente, tanto hospitalaria como extrahospitalaria, presenta un continuo y progresivo crecimiento, llegando a alcanzar en la actualidad unas cifras cercanas a la saturación del sistema, con 24,4 millones de urgencias hospitalarias en $2005^{1}$. Éste no es un problema exclusivamente nacional, sino que se produce en la mayoría de los países desarrollados, sin distinción de sus sistemas sanitarios. Junto a una tendencia creciente en su utilización y gran variabilidad en la demanda ${ }^{2,3}$ tanto en el sector hospitalario como a nivel extrahospitalario, existe la constatación de que una parte importante de los casos deberían ser atendidos en otros niveles asistenciales, con lo que se hace un uso inadecuado de estos servi$\operatorname{cios}^{4,5}$. Numerosos factores se han señalado para justificar dicho incremento, tanto por parte de la demanda (envejecimiento progresivo, incremento de las enfermedades crónicas, falta de cultura sanitaria, incorporación de la mujer al mercado de trabajo, aumento de la población, cultura de la inmediatez, nivel socioeconómico y cultural, etc.) como de la oferta (incremento de la oferta, accesibilidad geográfica, nivel de aseguramiento, facilidad de acceso al sistema etc.).

El uso inadecuado de estos servicios ${ }^{6,7}$ motiva una falta de continuidad asistencial, la falta de recepción de prácticas preventivas, un incremento de los costes asistenciales $^{8-10}$, medicalización y dificultad en la atención a las enfermedades realmente graves.

No existe una definición aceptada de lo que es una demanda apropiada de atención urgente, ni su reverso, o demanda inapropiada, lo que explica la gran variabilidad en su cuantificación según diferentes autores y países, con cifras que oscilan entre el 5 y el $86 \%$ de inadecuación cuando se utilizan criterios médicos ${ }^{11,12}$, lo que pone en evidencia la falta de consenso entre los profesionales sobre lo que constituye una urgencia médica. Empleando criterios objetivos mediante el Protocolo de Adecuación de las Urgencias Hospitalarias (PAUH) ${ }^{13}$ los autores sitúan la demanda inadecuada a urgencias en cifras del $30 \%{ }^{5,14}$, pero excluyen del estudio las urgencias pediátricas, de traumatología y obstétricas.

Los resultados en la demanda de asistencia extrahospitalaria parecen seguir una línea paralela a la demanda hospitalaria tanto en la frecuentación como a la inadecuación de la misma con cifras del $62 \%$ de inadecuación ${ }^{15}$.

Las numerosas propuestas e intervenciones dirigidas a reducir el aumento de frecuentación a las urgencias como facilitar la accesibilidad a la atención primaria, mejoras educativas, reformas organizativas, etc. han tenido una efectividad muy limita$\mathrm{da}^{16}$. Cabe pensar que una de las razones sea que se desconoce la opinión de los usuarios, los pacientes que demandan la urgencia, eslabón imprescindible y básico en la cadena de atención.

Las publicaciones, teniendo como sujeto activo el usuario o paciente, son limitadas. Se debe estudiar y analizar la percepción de la necesidad que determina la demanda de atención urgente porque lo que en el fondo subyace es determinar quién define lo que es urgente si el paciente o el sistema. O mejor, si la demanda es inadecuada o bien hay una oferta insuficiente.

El objetivo del trabajo es describir el perfil del demandante de urgencia hospitalaria y extrahospitalaria y conocer los factores que motivan la demanda de atención urgente.

\section{METODOLOGÍA}

Se realizó un estudio multicéntrico, transversal, descriptivo, circunscrito a las urgencias atendidas en el ámbito extrahospitalario de los Servicios Normales de Urgencias (SNU). Se invitó a todos los servicios de urgencias hospitalarios de los hospitales públicos de Navarra. Todos participaron excepto el Hospital Comarcal Reina Sofía de Tudela.

El total de tarjetas de identificación sanitaria (TIS) en Navarra a fecha de 30 de diciembre de 2007 es de 599.661. El número 
de TIS adscrita a los centros de estudio extrahospitalario es de 366.517 TIS y 507.138 TIS para el nivel hospitalario.

Se define como población de estudio todos los usuarios o demandantes de servicios médicos urgentes del SNU y servicio hospitalario de urgencias (SUH). Se excluye de la recogida de información a nivel hospitalario los niveles I y II en triaje previo (en 5 niveles de triaje) y las urgencias de obstetricia-ginecología. A nivel extrahospitalario se excluyen aquellos pacientes asimilables a los niveles I y II hospitalarios, urgencias visitadas en domicilio y accidentes de tráfico. También se excluyen para ambos niveles los demandantes exclusivos de servicios de enfermería.

Se diseña un cuestionario específico a partir de los diseñados por Sempere y $\operatorname{col}^{13}$ y Aranaz y col${ }^{7}$ y de reflexión propia, realizándose una validación de contenido mediante una prueba piloto previa. Las variables estudiadas son edad, sexo, país de nacimiento, autopercepción de gravedad (leve, moderada, grave), motivo de consulta, estado de salud habitual, utilización previa del servicio de urgencias, si fue por el mismo o por motivo diferente, razones que motivan la demanda y sintomatología que la motiva. Se han establecido un total de 18 razones de demanda, agrupadas para su análisis en 3 bloques. Cada bloque agrupa aquellas respuestas que a juicio de los autores son debidas básicamente a razones de preocupación y/o aparición de síntomas preocupantes (razones de necesidad), motivadas por la organización y gestión del sistema (razones de sistema) o bien por razones de comodidad y/o sociolaborales (razones de comodidad).

El cuestionario autorrellenable se entregó al paciente en el momento de recoger sus datos de filiación y fue cumplimentado por el propio enfermo (o por acompañante) el cual lo entregaba rellenado preferiblemente antes de realizar la consulta demandada. La pregunta referida a conocer las razones que le motivan a acudir a urgencias admite respuestas múltiples. $\mathrm{El}$ estudio se realizó en el periodo del 15 al 21 de noviembre de 2007.
A nivel hospitalario la recogida de información se realizó durante las 24 horas del día; a nivel extrahospitalario el horario de atención en SNU era de 15 a 8 horas para los días laborables y las 24 horas los días festivos. En el área de Pamplona, dada la extensión y demanda de la población, se incluyeron 3 centros periféricos de urgencias (CPU) con horario de atención más reducido.

Las urgencias de pediatría para el SNU de Pamplona únicamente se realizan hasta las 20 horas. A partir de este horario únicamente se contemplan a nivel hospitalario. En los demás SNU de Navarra las urgencias de pediatría son atendidas en todo el horario de atención de las urgencias.

Demandante de urgencia hospitalaria por iniciativa propia es todo demandante, excepto el paciente remitido por su médico de empresa o de cabecera o aquel que acude al hospital, después de acudir a urgencia extrahospitalaria, por recomendación médica.

Los análisis se realizan con los paquetes estadísticos Epiinfo. 6 y SPSS 15, calculándose Odds Ratio (OR) con su intervalo de confianza al 95\% (IC 95\%), la prueba de la t de Student, prueba de Ji cuadrado de tendencia lineal y análisis de residuos ajustados estandarizados en las tablas de contingencia. También se realizaron análisis univariantes y multivariantes, mediante regresión logística para analizar la asociación entre autopercepción de gravedad y acudir a hospital y las otras variables de estudio. El estudio no fue remitido a ningún comité de ética para su aprobación.

\section{RESULTADOS}

Sobre un total de 5.531 consultas demandadas se han recogido 2.364 cuestionarios cumplimentados, lo que representa el $42,7 \%$ del total.

Los síntomas que motivan el acudir a urgencias se han agrupado por aparatos (Tabla 1), representando los procesos respiratorios de vías altas y los del aparato locomotor el 44,3\% del total de atenciones. Existe una asociación entre el tipo de proceso y el servicio hospitalario o extrahospitalario utilizado $(\mathrm{p}<0,001)$. 
Tabla 1. Patología atendida en urgencias hospitalaria y extrahospitalaria según grupo diagnóstico. Navarra 2007 (número y porcentaje)

\begin{tabular}{lrrr}
\hline \multicolumn{1}{c}{ Procesos de salud } & \multicolumn{1}{c}{ Total } & \multicolumn{1}{c}{ SUH } & \multicolumn{1}{c}{ SNU } \\
\hline Procesos respiratorios de vías altas & $419(22,4)$ & $62(14,8)$ & $357(85,2)$ \\
Procesos oculares & $89(4,7)$ & $16(18)$ & $73(82)$ \\
Procesos del aparato locomotor & $410(21,9)$ & $213(52)$ & $197(48)$ \\
Procesos cardio-respiratorios & $122(6,5)$ & $73(59,8)$ & $49(40,2)$ \\
Procesos abdominales & $200(10,7)$ & $80(40)$ & $120(60)$ \\
Procesos dermatológicos & $87(4,6)$ & $15(17,2)$ & $72(82,8)$ \\
Procesos hepático-renales & $51(2,7)$ & $22(43,1)$ & $29(56,9)$ \\
Signos y síntomas mal definidos & $496(26,5)$ & $193(38,9)$ & $303(61,1)$ \\
Total & $1.874(100)$ & $674(36)$ & $1.200(64)$ \\
\hline
\end{tabular}

$\mathrm{p}<0,001^{*}$

${ }^{*} \mathrm{X}^{2}$ de Pearson. SNU: Servicio de urgencias extrahospitalarias. SUH: Servicio de urgencias hospitalarias.

Tabla 2. Características de los pacientes y factores que condicionan la demanda de atención urgente hospitalaria (SUH). Navarra 2007.

\begin{tabular}{lrrrrr}
\hline & & SUH & SNU & & \\
& $\mathbf{N}$ & $\mathbf{N}(\%)$ & $\mathbf{N}(\%)$ & OR (SUH) & IC 95\% \\
\hline Grupos de edad & $\mathbf{2 . 3 2 8}$ & & & & \\
$\quad<15$ años & 668 & $200(29,9)$ & $468(70,1)$ & 1 & \\
$\quad 15$ años & 1.660 & $669(40,3)$ & $991(59,7)$ & 1,6 & $1,3-1,9$ \\
\hline Sexo & $\mathbf{2 . 3 3 1}$ & & & & \\
$\quad 1.218$ & $411(33,7)$ & $807(66,3)$ & 1 & \\
$\quad$ Mujer & 1.113 & $463(41,6)$ & $650(58,4)$ & 1,4 & $1,2-1,7$ \\
$\quad$ Varón & 2.306 & & & & \\
\hline Lugar de nacimiento & 263 & $98(37,3)$ & $165(62,7)$ & 1 & \\
$\quad$ Extranjero & 2.043 & $768(37,6)$ & $1.275(62,4)$ & 1,01 & $0,8-1,3$ \\
$\quad$ España & $\mathbf{2 . 2 5 0}$ & & & & \\
\hline Estado de salud habitual & 2.023 & $718(35,5)$ & $1.305(64,5)$ & 1 & \\
$\quad$ Bueno & 227 & $118(52)$ & $109(48)$ & 2 & $1,5-2,6$ \\
$\quad$ Malo & $\mathbf{2 . 3 0 6}$ & & & & \\
\hline Mes anterior en urgencias & 1.869 & $707(37,8)$ & $1.162(62,2)$ & 1 & \\
$\quad$ No & 437 & $153(35)$ & $284(65)$ & 1,1 & $0,9-1,4$ \\
$\quad$ Sí & $\mathbf{4 3 7}$ & & & & \\
\hline Por el mismo motivo & 255 & $74(29)$ & $181(71)$ & 1 & \\
$\quad$ No & 182 & $79(43,4)$ & $103(56,6)$ & 1,9 & $1,3-2,8$ \\
$\quad$ Sí & $\mathbf{2 . 1 8 6}$ & & & & \\
\hline Impresión que me motiva acudir a urgencias & 527 & $126(23,9)$ & $401(76,1)$ & 1 & \\
Leve & 1.289 & $461(35,8)$ & $828(64,2)$ & 1,9 & $1,4-2,2$ \\
Moderada & 370 & $219(59,2)$ & $151(40,8)$ & 4,6 & $3,5-6,2$ \\
$\quad$ Grave & & & &
\end{tabular}

SUH: Servicio de urgencias hospitalarias

SNU: Servicio de urgencias extrahospitalarias

OR: Odds Ratio 
Por tipo de atención (SUH o SNU) (Tabla 2) se observan diferencias significativas respecto a edad de los pacientes, sexo varón, estado de salud habitual malo y haber acudido por el mismo motivo a urgencias en el mes anterior. El 29,9\% de los pacientes de menos de 15 años y el 40,3\% de los pacientes de 15 y más años acude a urgencias hospitalarias. Se ha detectado una asociación lineal entre la percepción de gravedad y el acudir al hospital $(\mathrm{p}<0,001)$. A mayor percepción de gravedad, aumenta la demanda en los servicios de urgencia hospitalarios.

La edad media de los usuarios para el total del estudio es de 31,6 años con un rango entre 0 y 93 años. Los usuarios del SUH tienen una edad media mayor (37,6 años) que los usuarios de SNU (28 años), siendo esta diferencia significativa $(\mathrm{p}<0,001)$.

Tabla 3. Demanda de atención urgente hospitalaria y extrahospitalaria según razones de demanda. Navarra 2007.

\begin{tabular}{lrrrrrrrr}
\hline & & & & SUH & SNU & SUH & SUH \\
& & N & \%* & n (\%) & n (\%) & OR & IC 95\% \\
\hline Bloque A-Por razones de necesidad & & & & & & & \\
\hline & Sí & 1.568 & 66,3 & $736(47)$ & $832(53)$ & 3,9 & $3,2-4,9$ \\
& No & 796 & 33,7 & $146(18,5)$ & $650(81,5)$ & 1 &
\end{tabular}

A1-Voy al SUH después de acudir a el SNU por consejo médico

$\begin{array}{rrrrrrr}\text { Sí } & 311 & 13,2 & 190(61) & 121(39) & \text { OR: } 3,1 & 2,4-4,0 \\ \text { No } & 2.053 & 86,8 & 692(33,5) & 1.361(66,5) & 1 & \end{array}$

A2-Dijeron fuera a urgencias si no mejoraba

$\begin{array}{rrrrrrr}\text { Sí } & 381 & 16,1 & 189(49,5) & 192(50,5) & \text { OR:1,8 } & 1,5-2,3 \\ \text { No } & 1.983 & 83,9 & 693(35) & 1.290(65) & 1 & \end{array}$

A3-El médico de cabecera no soluciona mis problemas

$\begin{array}{rrrrrrr}\text { Sí } & 115 & 4,9 & 63(55) & 52(45) & 2,1 & 1,5-3,1 \\ \text { No } & 2.249 & 95,1 & 819(36,5) & 1.430(63,5) & 1 & \end{array}$

A4-No tengo médico de cabecera y no se otro lugar donde acudir

$\begin{array}{rrrrrrr}\text { Sí } & 129 & 5,5 & 46(35,5) & 83(64,5) & 0,9 & 0,6-1,3 \\ \text { No } & 2.235 & 94,5 & 836(37,5) & 1.399(62,5) & 1 & \end{array}$

A5-Estoy preocupado, mi problema es serio y no puedo esperar

$\begin{array}{rrrrrrr}\text { Sí } & 1.042 & 44,1 & 450(43) & 592(57) & 1,6 & 1,3-1,9 \\ \text { No } & 1.322 & 55,9 & 432(32,5) & 890(67,5) & 1 & \end{array}$

A6-Me ha remitido el médico de empresa-cabecera

\begin{tabular}{lrrrrrrr} 
& Sí & 322 & 13,6 & $232(72)$ & $90(28)$ & 5,5 & $4,3-7,2$ \\
& No & 2.042 & 86,4 & $650(32)$ & $1.392(68)$ & 1 & \\
\hline Bloque B-Por razones de sistema & & & & & & & \\
\hline & Sí & 709 & 30 & $248(35)$ & $461(65)$ & 0,9 & $0,7-1,04$ \\
& No & 1.655 & 70 & $634(38,5)$ & $1.021(61,5)$ & 1 & \\
B1-Por mayor confianza en el hospital & & & & & & & \\
& Sí & 199 & 8,4 & $144(72,5)$ & $55(37,5)$ & 5,1 & $3,6-7,1$ \\
& No & 2.165 & 91,6 & $738(34)$ & $1.427(66)$ & 1 & \\
\hline
\end{tabular}




\begin{tabular}{ccccccc}
\hline & & SUH & SNU & SUH & SUH \\
& N & $\% *$ & n (\%) & n (\%) & OR & IC 95\% \\
\hline
\end{tabular}

B2-Por mayor tecnología diagnóstica

$\begin{array}{rrrrrrr}\text { Sí } & 186 & 7,9 & 139(74,5) & 47(25,5) & 5,7 & 4,1-8,0 \\ \text { No } & 2.178 & 92,1 & 743(34) & 1.435(66) & 1 & \end{array}$

B3-Hay consulta de pediatría por la tarde-noche

$\begin{array}{rrrrrrr}\text { Sí } & 214 & 9,1 & 53(25) & 161(75) & 0,5 & 0,4-0,7 \\ \text { No } & 2.150 & 90,9 & 829(38,5) & 1.321(61,5) & 1 & \end{array}$

B4-Pendiente de pruebas y por urgencias las adelanto

$\begin{array}{rrrrrrr}\text { Sí } & 37 & 1,6 & 25(67,5) & 12(32,5) & 3,6 & 1,8-7,1 \\ \text { No } & 2.327 & 98,4 & 857(37) & 1.470(63) & 1 & \end{array}$

B5-Medico de cabecera cita para varios días después

$\begin{array}{rrrrrrr}\text { Sí } & 365 & 15,4 & 64(17,5) & 301(82,5) & 0,3 & 0,2-0,4 \\ \text { No } & 1.999 & 84,6 & 818(41) & 1.181(59) & 1 & \end{array}$

B6-La lista de espera es enorme y no me llaman

\begin{tabular}{rrrrrrrr} 
& Sí & 91 & 3,9 & $40(44)$ & $51(56)$ & 1,3 & $0,9-2,0$ \\
& No & 2.273 & 96,1 & $842(37)$ & 1.431 & 1 & \\
\hline Bloque C-Por razones de comodidad & & & & & & & \\
\hline & Sí & 962 & 40,7 & $212(22)$ & $750(78)$ & 0,3 & $0,26-0,4$ \\
& No & 1.402 & 59,3 & $670(47,8)$ & $732(52,2)$ & 1 &
\end{tabular}

C1-Por comodidad, no tengo que pedir cita

$\begin{array}{rrrrrrr}\text { Sí } & 119 & 5 & 19(16) & 100(84) & 0,3 & 0,2-0,5 \\ \text { No } & 2.245 & 95 & 863(38,4) & 1.382 & 1 & \end{array}$

C2-Para conocer una segunda opinión

$\begin{array}{rrrrrrr}\text { Sí } & 67 & 2,8 & 33(49,3) & 34(50,7) & 1,7 & 1,02-2,7 \\ \text { No } & 2.297 & 97,2 & 849(37) & 1.448 & 1 & \end{array}$

C3-Por no perder horas de trabajo-escuela

$\begin{array}{rrrrrrr}\text { Sí } & 297 & 12,6 & 42(14,1) & 255(85,9) & 0,24 & 0,2-0,3 \\ \text { No } & 2.067 & 87,4 & 840(40,6) & 1.227 & 1 & \end{array}$

C4-Por no esperar al día siguiente

$\begin{array}{rrrrrrr}\text { Sí } & 465 & 19,7 & 81(17,4) & 384(82,6) & 0,29 & 0,22-0,4 \\ \text { No } & 1.899 & 80,3 & 801(42,2) & 1.098 & 1 & \end{array}$

C5-Me hacen diferentes pruebas de una vez, es más cómodo

$\begin{array}{rrrrrrr}\text { Sí } & 99 & 4,2 & 52(52,5) & 47(47,5) & 1,9 & 1,3-2,9 \\ \text { No } & 2.265 & 95,8 & 830(36,6) & 1.435 & 1 & \end{array}$

C6-Mi problema no es serio, pero si antes me tratan, antes me curo

\begin{tabular}{rrrrrrr} 
Sí & 369 & 15,6 & $85(23)$ & $284(77)$ & 0,45 & $0,3-0,6$ \\
No & 1.995 & 84,4 & $797(39,9)$ & $1.198(60,1)$ & 1 & \\
\hline
\end{tabular}

*: es el porcentaje de $\mathrm{N}$ respecto al total de cuestionarios. 
Entre las razones por las cuales se acude a urgencias (Tabla 3), el bloque de razones de necesidad es el más numeroso, señalado por el $66,3 \%$ de los encuestados. Quienes aducen estas razones presentan una probabilidad 4 veces superior de acudir a un SUH respecto a acudir a un SNU. Dentro de este bloque, el subgrupo más importante con el $66,5 \%$ señala "estoy preocupado, mi problema es serio y no puedo esperar". Para el total de encuestados, este subgrupo representa el 43,2\% (OR:1,6; IC:1,3-1,9). No se ha detectado relación entre la utilización de las urgencias hospitalarias y el bloque de razones de sistema, señaladas por el $30 \%$ del total de demandantes. Las razones de comodidad, señaladas por el 40,7 \% de los encuestados, representan un factor protector para acudir a un SUH (OR: 0,3; IC: 0,26-0,4).

Existe asociación entre la autopercepción de gravedad y las características de los demandantes (Tabla 4). El 29,1\% de los que acuden al SNU tienen una percepción leve, mientras que el $27,2 \%$ de los que acuden a urgencias hospitalarias señalan una autopercepción grave. Existe asociación entre el estado de salud habitual y la percepción de gravedad, siendo ésta más grave entre las personas con un estado habitual de salud malo.

Tabla 4. Autopercepción de gravedad de los demandantes de urgencias hospitalaria y extrahospitalaria y factores asociados. Navarra 2007.

\begin{tabular}{|c|c|c|c|c|c|c|}
\hline & $\mathbf{N}$ & $\%$ & $\begin{array}{l}\text { Leve } \\
\mathrm{N}(\%)\end{array}$ & $\begin{array}{c}\text { Moderada } \\
\text { N (\%) }\end{array}$ & $\begin{array}{l}\text { Grave } \\
\mathrm{N}(\%)\end{array}$ & $\mathbf{P}^{*}$ \\
\hline Grupos de edad & 2.164 & & & & & \\
\hline$<15$ años & 632 & 29 & $182(28,8)$ & $395(62,5)$ & $55(8,7)$ & $<0,001$ \\
\hline$\geq 15$ años & 1.532 & 71 & $338(22,1)$ & $883(57,6)$ & $311(20,3)$ & \\
\hline Sexo & 2.166 & & & & & \\
\hline Mujer & 1.129 & 52 & $273(24,2)$ & $684(60,6)$ & $172(15,2)$ & 0,07 \\
\hline Varón & 1.037 & 48 & $247(23,8)$ & $594(57,3)$ & $1.196(8,9)$ & \\
\hline Lugar de nacimiento & 2.144 & & & & & \\
\hline Extranjero & 232 & 11 & $47(20,3)$ & $135(58,2)$ & $50(21,6)$ & 0,07 \\
\hline España & 1.912 & 89 & $472(24,7)$ & $1.130(59,1)$ & $310(16,2)$ & \\
\hline Estado de salud habitual & 2.196 & & & & & \\
\hline Bueno & 1.887 & 90 & $487(25,8)$ & $1.120(59,4)$ & $280(14,8)$ & $<0,001$ \\
\hline Malo & 209 & 10 & $24(11,5)$ & $113(54,1)$ & $72(34,4)$ & \\
\hline Mes anterior en urgencias & 2.148 & & & & & \\
\hline No & 1.742 & 81 & $426(24,5)$ & $1.041(59,8)$ & $275(15,8)$ & 0,023 \\
\hline Sí & 406 & 19 & $91(22,4)$ & $228(56,2)$ & $87(21,4)$ & \\
\hline $\begin{array}{l}\text { Motivo por el que acude respecto al } \\
\text { mes anterior }\end{array}$ & 437 & & & & & \\
\hline Por el mismo motivo & 172 & 42,4 & $33(19,2)$ & $93(54,1)$ & $46(26,7)$ & 0,06 \\
\hline Por otro motivo & 234 & 57,6 & $58(24,8)$ & $135(57,7)$ & $41(17,5)$ & \\
\hline Centro donde solicitó la atención & 2.186 & & & & & \\
\hline SUH & 806 & 37 & $126(15,6)$ & $461(57,2)$ & $219(27,2)$ & $<0,001$ \\
\hline SNU & 1.380 & 63 & $401(29,1)$ & $828(60)$ & $151(10,9)$ & \\
\hline
\end{tabular}

* $\mathrm{X}^{2}$ de Pearson

SUH: servicio de urgencias hospitalario

SNU: urgencias extrahospitalarias 
La percepción de gravedad y las razones por las que se acude a urgencias (Tabla 5), se observa asociación estadísticamente significativa para razones de necesidad, razones de sistema y razones de comodidad.
Se observa asociación lineal entre razones de necesidad y sensación de gravedad moderada y grave $(p<0,001)$ y entre razones de comodidad y sensación de gravedad leve $(\mathrm{p}<0,001)$.

Tabla 5. Autopercepción de gravedad señaladas por los demandantes de urgencias hospitalarias y extrahospitalarias según razones de demanda. Navarra 2007.

\begin{tabular}{rrrrrrrr}
\hline & & & \multicolumn{2}{c}{$\begin{array}{c}\text { Leve } \\
\text { n (\%) }\end{array}$} & $\begin{array}{c}\text { Moderada } \\
\text { n (\%) }\end{array}$ & \multicolumn{1}{c}{$\begin{array}{c}\text { Grave } \\
\text { n (\%) }\end{array}$} & P $^{*}$ \\
\hline Bloque A-Por razones de necesidad & & & & & & \\
\hline Sí & $\mathbf{1 . 4 4 9}$ & $\mathbf{6 6 , 3}$ & $242(16,7)$ & $891(61,5)$ & $316(21,8)$ & $<0,001$ \\
No & $\mathbf{7 3 7}$ & $\mathbf{3 3 , 7}$ & $285(38,7)$ & $398(54)$ & $54(7,3)$ &
\end{tabular}

A1-Voy al SUH después de acudir a el SNU por consejo médico

$\begin{array}{rrrrrrr}\text { Sí } & 290 & 13,3 & 37(12,8) & 183(63,1) & 70(24,1) & <0,001 \\ \text { No } & 1896 & 86,7 & 490(25,8) & 1.106(58,3) & 300(15,8) & \end{array}$

A2-Dijeron fuera a urgencias si no mejoraba

$\begin{array}{rrrrrrr}\text { Sí } & 354 & 16,2 & 58(16,4) & 202(57,1) & 94(26,6) & <0,001 \\ \text { No } & 1.832 & 83,8 & 469(25,6) & 1.087(59,3) & 276(15,1) & \end{array}$

A3-El médico de cabecera no soluciona mis problemas

$\begin{array}{rrrrrrr}\text { Sí } & 108 & 4,9 & 12(11,1) & 68(63) & 28(25,9) & 0,001 \\ \text { No } & 2.078 & 95,1 & 515(24,8) & 1.221(58,8) & 342(16,5) & \end{array}$

A4-No tengo médico de cabecera y no se otro lugar donde acudir

$\begin{array}{rrrrrrr}\text { Sí } & 115 & 5,3 & 28(24,3) & 77(67) & 10(8,7) & 0,046 \\ \text { No } & 2.071 & 94,7 & 499(24,1) & 1.212(58,5) & 360(17,4) & \end{array}$

A5-Estoy preocupado, mi problema es serio y no puedo esperar

$\begin{array}{rrrrrrr}\text { Sí } & 975 & 44,6 & 124(12,7) & 605(62,1) & 246(25,2) & <0,001 \\ \text { No } & 1.211 & 55,4 & 403(33,3) & 684(56,5) & 124(10,2) & \end{array}$

A6-Me ha remitido el médico de empresa-cabecera

\begin{tabular}{rrrrrrrr} 
Sí & 297 & 13,6 & $48(16,2)$ & $166(55,9)$ & $83(27,9)$ & $<0,001$ \\
No & 1.889 & 86,4 & $479(25,4)$ & $1.123(59,4)$ & $287(15,2)$ & \\
\hline
\end{tabular}

Bloque B-Por razones de sistema

$\begin{array}{rrrrrrr}\text { Sí } & \mathbf{6 6 6} & \mathbf{3 0 , 5} & 138(20,7) & 431(64,7) & 97(14,6) & 0,001 \\ \text { No } & \mathbf{1 . 5 2 0} & \mathbf{6 9 , 5} & 389(25,6) & 858(56,4) & 273(18) & \end{array}$

B1-Por mayor confianza en el hospital

$\begin{array}{rrrrrrr}\text { Sí } & 183 & 8,4 & 27(14,8) & 112(61,2) & 44(24) & 0,001 \\ \text { No } & 2.003 & 91,6 & 500(25) & 1.177(58,8) & 326(16,3) & \end{array}$

B2-Por mayor tecnología diagnóstica

$\begin{array}{rrrrrrr}\text { Sí } & 171 & 7,8 & 29(17) & 112(65,5) & 30(17,5) & 0,07 \\ \text { No } & 2.015 & 92,2 & 498(24,7) & 1.177(58,4) & 340(16,9) & \end{array}$

B3-Hay consulta de pediatría por la tarde-noche

\begin{tabular}{rrrrrrr} 
Sí & 203 & 9,3 & $39(19,2)$ & $133(65,5)$ & $31(15,3)$ & 0,12 \\
No & 1.983 & 90,7 & $488(24,6)$ & $1.156(58,3)$ & $339(17,1)$ & \\
\hline
\end{tabular}




\begin{tabular}{|c|c|c|c|c|c|c|}
\hline & $\mathbf{N}$ & $(\%) * *$ & $\begin{array}{l}\text { Leve } \\
\text { n (\%) }\end{array}$ & $\begin{array}{c}\text { Moderada } \\
\text { n (\%) }\end{array}$ & $\begin{array}{l}\text { Grave } \\
\text { n (\%) }\end{array}$ & $\mathbf{P}^{*}$ \\
\hline \multicolumn{7}{|c|}{ B4-Pendiente de pruebas y por urgencias las adelanto } \\
\hline Sí & 34 & 1,6 & $5(14,7)$ & $20(58,8)$ & $9(26,5)$ & 0,21 \\
\hline No & 2.152 & 98,4 & $522(24,3)$ & $1.269(59)$ & $361(16,8)$ & \\
\hline
\end{tabular}

B5-Medico de cabecera cita para varios días después

$\begin{array}{rrrrrrr}\text { Sí } & 344 & 15,7 & 82(23,8) & 227(66) & 35(10,2) & 0,001 \\ \text { No } & 1.842 & 84,3 & 445(24,2) & 1.062(57,7) & 335(18,2) & \end{array}$

B6-La lista de espera es enorme y no me llaman

\begin{tabular}{rrrrrrrr} 
Sí & 85 & 3,9 & $19(22,4)$ & $55(64,7)$ & $11(12,9)$ & 0,5 \\
No & 2.101 & 96,1 & $508(24,2)$ & $1.234(58,7)$ & $359(17,1)$ & \\
\hline Bloque C-Por razones de comodidad & & & & & & \\
\hline Sí & $\mathbf{9 0 6}$ & $\mathbf{4 1 , 4}$ & $295(32,6)$ & $511(56,4)$ & $100(11)$ & $<0,001$ \\
No & $\mathbf{1 . 2 8 0}$ & $\mathbf{5 8 , 6}$ & $232(18,1)$ & $778(60,8)$ & $270(21,1)$ & \\
C1-Por comodidad, no tengo que pedir cita & & & & & & \\
Sí & 112 & 5,1 & $39(34,8)$ & $60(53,6)$ & $13(11,6)$ & 0,02 \\
No & 2.074 & 94,9 & $488(23,5)$ & $1.229(59.3)$ & $357(17,2)$ &
\end{tabular}

C2-Para conocer una segunda opinión

$\begin{array}{rrrrrrr}\text { Sí } & 62 & 2,8 & 14(22,6) & 33(53,2) & 15(24,2) & 0,3 \\ \text { No } & 2.124 & 97,2 & 513(24,2) & 1.256(59,1) & 355(16,7) & \end{array}$

C3-Por no perder horas de trabajo-escuela

$\begin{array}{rrrrrrr}\text { Sí } & 282 & 12,9 & 85(30,1) & 164(58,2) & 33(11,7) & 0,006 \\ \text { No } & 1.904 & 87,1 & 442(23,2) & 1.125(59,1) & 337(17,7) & \end{array}$

C4-Por no esperar al día siguiente

$$
\begin{array}{rrrrrrr}
\text { Sí } & 436 & 19,9 & 151(34,6) & 238(54,6) & 47(10,8) & <0,001 \\
\text { No } & 1.750 & 80,1 & 376(21,5) & 1.051(60,1) & 323(18,5) &
\end{array}
$$

C5-Me hacen diferentes pruebas de una vez, es más cómodo

$$
\begin{array}{rrrrrrr}
\text { Sí } & 91 & 4,2 & 14(15,4) & 59(64,8) & 18(19,8) & 0,14 \\
\text { No } & 2.095 & 95,8 & 513(24,5) & 1.230(58,7) & 352(16,8) &
\end{array}
$$

C6-Mi problema no es serio, pero si antes me tratan, antes me curo

\begin{tabular}{rrrrrrr} 
Sí & 348 & 15,9 & $128(36,8)$ & $194(55,7)$ & $26(7,5)$ & $<0,001$ \\
No & 1.838 & 84,1 & $399(21,7)$ & $1.095(59,6)$ & $344(18,7)$ & \\
\hline
\end{tabular}

**: Corresponde al porcentaje de $\mathrm{N}$ respecto al total de cuestionarios (2.186) que presenta esta información.

En la distribución de la demanda (Fig. 1), se observa predominio de las razones de necesidad.

En el análisis multivariante (Tabla 6), el acudir a urgencias por el mismo motivo, razones de necesidad y razones de comodidad fueron predictores independientes de la demanda de urgencias hospitalarias. Quienes señalen razones de comodidad acuden menos a los SUH (OR:0,2; IC:0,1-0,4) mientras que quienes señalan razones de necesidad acuden mas a urgencias hospitalarias (OR:2,6; IC:1,5-4,8).

Quienes perciben su problema como leve (Tabla 7), acuden con más frecuencia a un SNU y presentan un estado de salud habitual bueno (OR:2,1; IC:1,3-3,2). Estos pacientes señalan las razones de comodidad (OR:1,6; IC:1,3-2,0) como las principales determinantes de la demanda. 


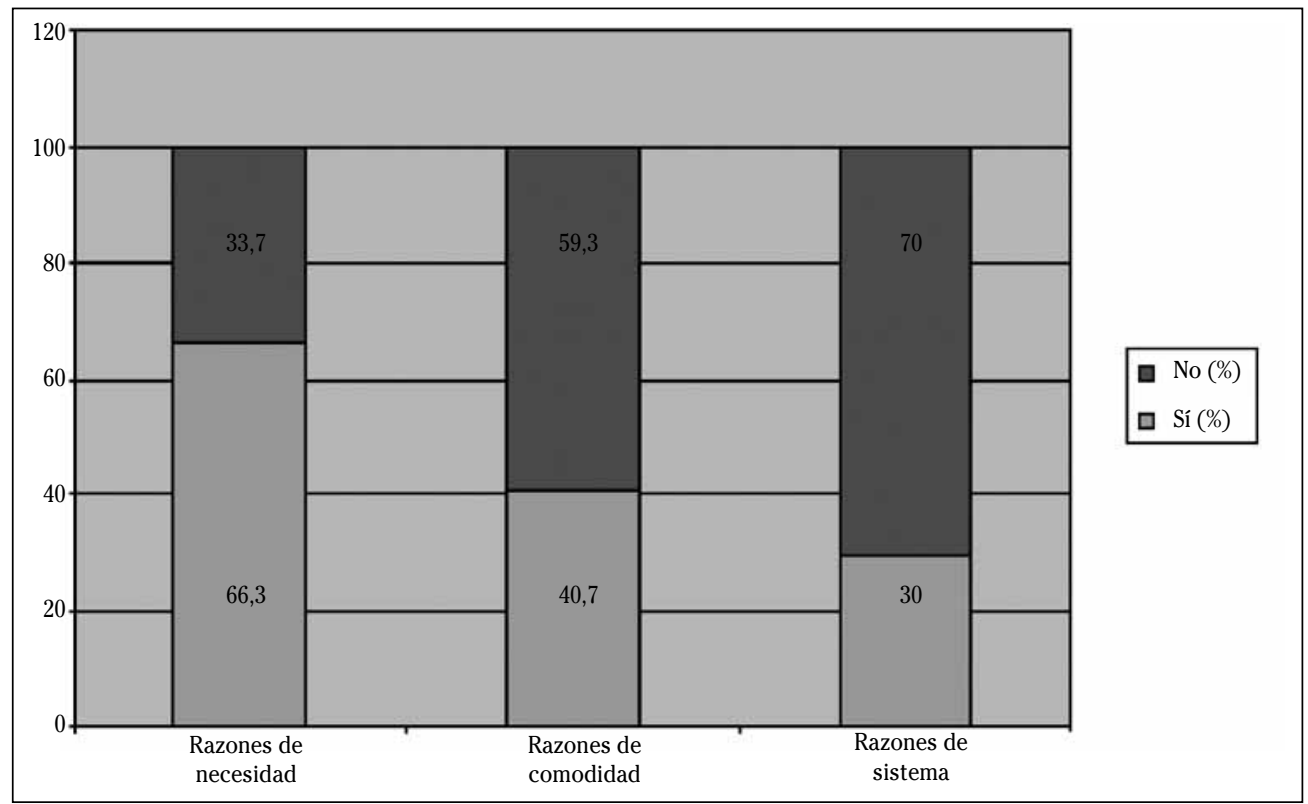

Figura 1. Solicitud de atención urgente. Razones de demanda. Navarra 2007.

Tabla 6. Demanda de urgencias hospitalarias (SUH) en Navarra según características de los pacientes ajustada por edad y sexo. 2007. Regresión logística.

\begin{tabular}{lcccccc}
\multicolumn{1}{c}{ Factor } & $\mathbf{N}$ & $\mathbf{\%}$ & ORb & IC95\% & ORa & IC 95\% \\
\hline $\begin{array}{l}\text { Impresión de gravedad } \\
\quad \text { Leve }\end{array}$ & 126 & 23,9 & 1 & & & \\
$\quad$ Moderada & 461 & 35,8 & 1,8 & $1,4-2,3$ & 1 & $0,5-1,9$ \\
$\quad$ Grave & 219 & 59,2 & 4,6 & $3,4-6,2$ & 1,6 & $0,8-3,4$ \\
Estado de salud habitual & & & & & & \\
$\quad$ Bueno & 718 & 35,5 & 1 & & & \\
$\quad$ Malo & 118 & 52 & 1,97 & $1,5-2,6$ & 1,4 & $0,8-2,4$ \\
Acudo a urgencias por & & & & & & \\
$\quad$ Mismo motivo & 182 & 43,4 & 1,9 & $1,3-2,8$ & 1,7 & $1,1-2,8$ \\
$\quad$ Otro motivo & 255 & 29 & 1 & & & \\
Razones de necesidad & & & & & & \\
$\quad$ Sí & 736 & 46,9 & 3,9 & $3,2-4,8$ & 2,6 & $1,5-4,8$ \\
$\quad$ No & 146 & 18,3 & 1 & & & \\
Razones de sistema & & & & & & \\
$\quad$ Sí & 248 & 35 & 0,87 & $0,7-1,1$ & 1,3 & $0,8-2,2$ \\
$\quad$ No & 634 & 38,3 & 1 & & & \\
Razones de comodidad & & & & & & \\
$\quad$ Sí & 212 & 22 & 0,31 & $0,3-0,4$ & 0,2 & $0,1-0,4$ \\
No & 670 & 47,8 & 1 & & & \\
\hline
\end{tabular}

ORb: Odds ratio bruta

ORa: Odds ratio ajustada

IC 95\%: Intérvalo de confianza al 95\% 
Tabla 7. Demanda de urgencias autopercibidas como LEVES según características de los demandantes ajustado por edad y sexo. Navarra 2007. Regresión logística.

\begin{tabular}{lcccccc}
\hline \multicolumn{1}{c}{ Factor } & $\mathbf{N}$ & $\mathbf{\%}$ & ORb & IC 95\% & ORa & IC 95\% \\
\hline $\begin{array}{l}\text { Acudo a urgencias de } \\
\quad \text { SUH }\end{array}$ & 126 & 15,6 & 1 & & & \\
$\quad$ SNU & 401 & 29,1 & 2,2 & $1,8-2,8$ & 1,4 & $1,1-1,9$ \\
Estado de salud habitual & & & & & & \\
$\quad$ Bueno & 487 & 25,8 & 2,7 & $1,7-4,3$ & 2,1 & $1,3-3,2$ \\
$\quad$ Malo & 24 & 11,5 & 1 & & & \\
Razones de necesidad & & & & & & \\
$\quad$ Sí & 242 & 16,7 & 0,4 & $0,3-0,4$ & 0,4 & $0,3-0,5$ \\
$\quad$ No & 285 & 38,7 & 1 & & & \\
Razones de sistema & & & & & & \\
$\quad$ Sí & 138 & 20,7 & 0,8 & $0,6-0,95$ & 0,6 & $0,5-0,8$ \\
$\quad$ No & 389 & 25,6 & 1 & & & \\
Razones de comodidad & & & & & & \\
Sí & 295 & 32,6 & 2,2 & $1,8-2,7$ & 1,6 & $1,3-2,0$ \\
No & 232 & 18,1 & 1 & & & \\
\hline
\end{tabular}

ORb: Odds Ratio bruta

ORa: Odds Ratio ajustada

IC $95 \%$ : intervalo de confianza al 95\%

\section{DISCUSIÓN}

El periodo temporal de recogida de información fue típico en cuanto a la situación epidemiológica, no existiendo ninguna situación epidemiológica que condicionara el tipo y volumen de la demanda de solicitud urgente.

El triaje en hospitales es realizado por enfermería, utilizando como criterio la presunción de gravedad. En SNU no se realiza triaje previo. La equiparación a los niveles I y II de triaje hospitalario y, por tanto, la urgencia en la atención y la inclusión o no en el estudio, está motivada por la impresión del administrativo y la comunicación previa al personal médico. Parece básica la necesidad de un equipo de profesionales, que decida las prioridades de asistencia sanitaria y la aplicación de un modelo de triaje estructurado, válido, útil y reproducible, de gran ayuda en la gestión de la asistencia urgente $^{17,18}$.

Los tres grandes grupos diagnósticos corresponden a procesos respiratorios de vías altas, procesos del aparato locomotor y signos y síntomas mal definidos, resultados similares a otros estudios ${ }^{19}$.

El autodiagnóstico es el determinante de la necesidad de solicitar atención urgente y del tipo de atención ${ }^{20}$. El conocimiento de la oferta de servicios, junto con el factor tiempo y las características de las actividades cotidianas del individuo o su situación vital, son los determinantes del tipo de atención solicitada. Cuanta más importancia tiene para el individuo el factor tiempo, más probabilidad existe que acuda a un $\mathrm{SUH}^{21}$.

Los factores de índole social, familiar y laboral tienen una importancia esencial a la hora de movilizar a los pacientes a acudir por propia iniciativa a los servicios de urgencias $^{22}$. La cuestión para el usuario es reducir la incertidumbre ${ }^{23,24}$ frente a una percepción de necesidad derivada de un problema de salud. Cuando el paciente elabora un autodiagnóstico claro (con síntomas ya conocidos) no se otorga el carácter de gravedad y se decide acudir a un ser- 
vicio u otro según el conocimiento de la oferta de servicios y de la situación vital del paciente ${ }^{21}$.

Más de la mitad de los pacientes incluidos en el bloque razones de sistema aducen "el médico le da hora para varios días después", problema factible de solución con una actuación y gestión eficiente sobre aquellos puntos donde el retraso en la atención primaria sea más acuciante.

Los demandantes que aducen razones de comodidad acuden más a un SNU, con edades entre 10 a 24 años y con sintomatología encuadrada principalmente en procesos respiratorios de vías altas. Es necesario considerar la importancia de la población flotante, universitaria, que posiblemente no tenga médico de cabecera y señale las razones escolares como las motivantes de la demanda.

En nuestro estudio, la población demanda más los servicios de urgencia extrahospitalarios fundamentalmente hasta la edad de 45 años, resultados contrarios a los observados en otros estudios ${ }^{19,25}$. No se ha detectado grado de desconocimiento de la oferta de servicios de urgencias extrahospitalarios ${ }^{26}$ como justificación del uso de las urgencias hospitalarias, ni tampoco de la existencia de barreras de acceso a estos servicios ${ }^{27}$. Los usuarios no utilizan las urgencias como puerta de entrada al sistema sanitario hospitalario, aún cuando sí pueda utilizarse como sustitutivo de la atención primaria ${ }^{27,28}$.

La edad media de los demandantes es inferior a la observada en otros estudios ${ }^{7,29}$, siendo mayor en las urgencias hospitalarias.

$\mathrm{El}$ alto porcentaje de pacientes que en el mes anterior acudieron a urgencias, por el mismo motivo, puede reflejar una escasa capacidad resolutiva de las urgencias a la vez que un buen trato en la atención y el conocimiento de los servicios por experiencias previas. Otros motivos señalados pueden ser la demora en otros niveles asistenciales y la probable cronicidad del proceso o la pluripatología del mismo, que requiere varias consultas ${ }^{30}$. Esto ha inducido a señalar la necesidad de un cambio de actitud de estos enfermos para reducir las consultas a urgencias y sustituirlas por el uso de dispositivos alternativos para conseguir un cambio cualitativo de la deman$\mathrm{da}^{30}$.

La proporción de encuestados que califican su proceso como leve $(24,1 \%)$ es inferior a la observada en otros estudios ${ }^{23,24,26,31}$ en los que la demanda está considerada exclusivamente desde el punto de vista médico y reducida a las urgencias hospitalarias; por el contrario, en nuestro estudio es el propio enfermo quien reconoce como tal la levedad del proceso. De este total, 1 de cada 4 pacientes acuden a un SUH. Si eliminamos el concepto de autopercepción moderada, el $58,8 \%$ de los pacientes se clasificaría con percepción leve.

La elevada proporción de pacientes con una percepción de gravedad leve es indicativa de una mala utilización de los servicios de atención primaria. Otros factores señalados en la bibliografía ${ }^{20}$ son el ser varones, de clases profesionales bajas y que muestran insatisfacción con su médico de cabecera junto con posibles factores extrasanitarios (laborales, familiares). Se han señalado varios factores en este uso perverso de los servicios de urgencias como es el evitar listas de espera o realizar actividades programadas diferidas, datos no señalados en nuestro estudio.

Algunos autores señalan que dada la escasa repercusión que las urgencias inapropiadas, de percepción leve, tienen en el normal funcionamiento de los $\mathrm{SUH}^{11}$ se ha señalado la necesidad de la Administración de adecuar la oferta de los $\mathrm{SUH}$, SNU y de camas de hospitalización sociosanitaria, de larga estancia, crónicos, etc. a la verdadera demanda ${ }^{11}$, lo cual entra en contradicción con los postulados que rigen la actuación en Atención Primaria.

El 64\% de los demandantes de urgencias hospitalarias lo hacen por iniciativa propia, cifra ligeramente inferior a la observada en otros estudios ${ }^{7,33}$.

Un factor señalado en la bibliografía hace referencia al mantenimiento de una atención primaria poco adecuada a las necesidades de salud actuales ${ }^{34}$. La oferta de servicios de salud debería ser el resultado 
de un análisis donde confluyan la opinión de los técnicos, las características epidemiológicas y demográficas y las necesidades expresadas por la población, debiendo incorporar estas en el proceso de elaboración de políticas de salud. La necesidad sentida tiene una gran correlación con la demanda de asistencia y la decisión de acceder al sistema sanitario. Algunos autores $^{34}$ señalan la necesidad de incorporar la realidad al sistema de salud, reorganizar los servicios de urgencias, buscando un equilibrio con la demanda real, en lugar de adecuar la demanda de la población a los servicios de urgencias.

La metodología utilizada en este estudio difiere respecto a otros estudios. Al ser un cuestionario autorrellenable por el paciente, se ha eliminado el sesgo de entrevistador y se ha conseguido una mayor libertad y realidad en su cumplimentación. Por el contrario, ha podido haber sesgos en la interpretación y delimitación de la pregunta por el paciente, así como, dada la tasa de emigrantes y su posible dificultad con el idioma, una mayor no respuesta en este colectivo.

Se han realizado multitud de intervenciones para reducir la utilización de los servicios de urgencias, fundamentalmente hospitalarios, como creación de centros de urgencias intermedios, sistemas de cribado, ampliación en la capacidad de la ofer$\mathrm{ta}^{24,25}$, establecimiento de normas capaces de cumplirse, encaminadas a disminuir el numero de usuarios que acuden por iniciativa propia ${ }^{26}$ y todo ello con resultados no satisfactorios.

En conclusión, un mejor conocimiento de la opinión y actitud de los usuarios puede delimitar la intervención a seguir. Los médicos deben participar en el debate, al igual que los usuarios, para intentar incidir en el mal uso de los servicios sanitarios, tanto por defecto como por exceso.

\section{Agradecimientos}

A todos aquellos, personal sanitario y administrativo que, con su ayuda, han hecho posible la recogida de información

\section{BIBLIOGRAFÍA}

1. Estadística de establecimientos sanitarios con régimen de internado, 2005. Instituto de Información Sanitaria, Ministerio de Sanidad y Consumo, 2007.

2. Ministerio de Sanidad y Consumo. Estadística de Establecimientos Sanitarios con Régimen de Internado. Datos básicos de la salud y los servicios sanitarios en España 2003. Disponible en: http://www.msc.es/estadEstudios/estadisticas/docs/Datos_basicos_salud.pdf (consultado 10-9-2009).

3. Millá J. Urgencias médicas: algo más que una serie televisiva. Med Clin (Barc) 2001; 117: 295-296.

4. Aranaz JM, Martínez R, Rodrigo V, Gómez F, ANTón P. Adecuación de la demanda de atención sanitaria en servicios de urgencias hospitalarios. Med Clin (Barc) 2004; 123: 615-618.

5. Lee A, Hazlett CB, Chow S, Law FL, Kam CW, Wong $\mathrm{P}$ et al. How to minimize inappropriate utilization of accident and emergency departments: improve the validity of classifying the general practice cases amongst the A\&E attendees. Health Policy 2003; 66: 159168 .

6. Tudela P, Modal JM. Urgencias hospitalarias. Med Clin (Barc) 2003; 120: 711-716.

7. Aranaz Andrés JM, Martínez Nogueras R, Gea Velázquez de Castro MT, Rodrigo Bartual V, Antón García P, Gómez Pajares F ¿Por qué los pacientes utilizan los servicios de urgencias hospitalarios por iniciativa propia? Gac Sanit 2006; 20: 311-315.

8. Pérez I, Gulllén F. Radiología innecesaria en Atención Primaria. An Sist Sanit Navar 2007; 30: 53-60.

9. Cunningham PJ, Clancy CM, Cohen JW, Wilets M. The use of hospital emergency departments for nonurgent health problems: a national perspective. Med Care Res Rev 1995; 52: 453474.

10. WiLLIAM RM. The cost of visits to emergency departments. N Engl J Med 1996; 334: 642646.

11. SÁnchez M. ¿Urgencias inadecuadas u oferta insuficiente? Med Clin (Barc) 2004; 123: 619620.

12. Cantero Hinojosa J, Sánchez-Cantalejo Ramírez E, Martínez Olmos J, Maeso Villafaña J, RodríGUEZ JimÉnez JJ, PRIETo Rodríguez MA et al. Inadecuación de las visitas a un servicio de urgencias hospitalario y factores asociados. Aten Primaria 2001; 28: 326-332. 
13. Sempere T, Peiró S, Sendra P, Martinez C, López I. Validez del Protocolo de Adecuación de Urgencias Hospitalarias. Rev Esp Salud Pública 1999; 73: 465-479.

14. Sempere-Selva T, Peiró S, Sendra-Pina P, MartinezEsPin C, LóPEz-Aguilera I. Inappropriate use of an accident and emergency department: magnitude, associated factors, and reasons- an approach with explicit criteria. Ann Emerg Med 2001; 37: 568-579.

15. Grupo de trabajo de la Sociedad Andaluza de MeDICINA FAMILIAR y Comunitaria. Ordenación de las urgencias en Atención Primaria. Aten Primaria 1992; 9: 269-275.

16. Miró O, Salgado E, Espinosa G, Estrada C, Martí C, CAMP J et al. Derivación sin visita desde los servicios de urgencia hospitalarios: cuantificación, riesgos y grado de satisfacción. Med Clin (Barc) 2006; 126: 88-93.

17. Gómez Jiménez J. Clasificación de pacientes en los servicios de urgencias y emergencias: Hacia un modelo de triaje estructurado de urgencias y emergencias. Emergencias 2003; 15: 165-174.

18. Aranguren E, Capel Ja, Solano M, Jean Louis C, Larumbe J, Elejalde J. Estudio de la validez pronóstica de la recepción, acogida y clasificación en el área de urgencias en un hospital terciario. An Sist Sanit Navar 2005; 28: 177188.

19. Torné Vilagrasa E, Guarga Rojas A, Torras Boatella MG, Pozuelo García A, Pasarín Rua M, BoRREll Thió C. Análisis de la demanda en los servicios de urgencia de Barcelona. Aten Primaria 2003; 32: 423-429.

20. MuRPhy AW. "Inappropiate» attenders at accident and emergency departments: definition, incidence and reasons for attendance (i-ii). Fam Pract 1998; 15: 23-32, 33-37.

21. Pasarín IM, Fernández de Sanmamed MJ, Calafell J, Borrell C, Rodríguez D, Campasol S et al. Razones para acudir a los servicios de urgencias hospitalarios. La población opina. Gac Sanit 2006; 20: 91-99.

22. Braun T, García Castrillo-Riesgo L, Krafft T, Díaz-Regañón Vilches G. Frecuentación del servicio de urgencias y factores sociodemográfica. Gac Sanit 2002; 16: 139-144.
23. Ochoa FJ, Ramalle-Gómara E, Villar A, Ruiz J, Bragado L, Gimeno C. Visitas inapropiadas al servicio de urgencias de un hospital general. Med Clin (Barc) 2000; 115: 377-378.

24. Castells X. Comentario. Utilización de los servicios de urgencias hospitalarios. La importancia de la perspectiva de los usuarios. Gac Sanit 2006; 20: 99-100.

25. Núñez S, Martínez-Sanz R, OJeda E, Aguirre-Jaime A. Perfil clínico-asistencial e impacto del retorno inesperado a urgencias de un mayor de 65 años. An Sist Sanit Navar 2006; 29: 199-206.

26. Antón M, Peña J, Santos R, Sempere E, Martínez J, PÉrula LA. Demanda inadecuada a un servicio de urgencias pediátrico hospitalario: factores implicados. Med Clin (Barc) 1992; 99: 743-746.

27. Baker DW, Stevens CD, Brook RH. Determinants of emergency department use by ambulatory patients at an urban public hospital. Ann Emerg Med 1995; 25: 311-316.

28. MenÉndez JM. Gestión de las urgencias. Organización y gestión de la atención urgente extrahospitalaria. En: Del Llano J, Ortún V, Martín JM, Gené J. Gestión Sanitaria. Innovaciones o desafíos. Barcelona: Masson, 1998, p 403-418.

29. Vázquez B, Pardo G, Fernández G, Canals M, DelGado M, Navas M. ¿Por qué acuden nuestros pacientes a urgencias del hospital? Aten Primaria 2000; 25: 98-105.

30. Domingo-Ribas C, Ortún-Rubio V. Urgencias hospitalarias o el colapso crónico: Los pacientes crónicos no deberían colapsar urgencias. Arch Bronconeumol 2006; 42: 257-259.

31. Peiró S, Sempere Selva T, Oterino de la Fuente D. Efectividad de las intervenciones para reducir la utilización inapropiada de los servicios hospitalarios de urgencias. Revisando la literatura 10 años después del informe del Defensor del Pueblo. Economía y Salud 1999; 33: 3-12.

32. Defensor del Pueblo. Informe sobre Urgencias Hospitalarias. Oficina del Defensor del Pueblo, Madrid, 1988.

33. Moreno MilLán E. ¿̨Y si adaptáramos los servicios hospitalarios de urgencias a la demanda social y no a las necesidades de salud? Emergencias 2008; 20: 276-284. 